\title{
Color Image Segmentation Using Improved Region Growing and K-Means Method
}

\author{
Kewal Krishan, Sukhjit Singh \\ kewal86kamboj@gmail.com,sukhjit_singh@rediffmail.com \\ Department of Electronic and Communication Engineering \\ GTBKIET Chhapianwali, Malout(Punjab), India
}

\begin{abstract}
In areas such as computer vision and image processing, image segmentation has been and still is a relevant research area due to its wide spread usage and application. The traditional segmentation technique which is used in gray-scale mathematical morphology is watershed transform. Region Growing is an approach to image segmentation in which neighbouring pixels are examined and added to a region class if no edges are detected. This process is iterated for each boundary pixel in the region. In this paper, we made enhancements in watershed algorithm and region growing algorithm for image and color segmentation. The new enhanced algorithm is implemented in MATLAB and results are compared with the existing technique in the form of visualization and on the basis of Liu's F- factor values.
\end{abstract}

Keywords: - Color Segmentation, Image Segmentation, Watershed, Liu's F-factor, region growing

\section{INTRODUCTION}

Image segmentation is the process of dividing the given image into regions homogenous with respect to certain features, and which hopefully correspond to real objects in the actual scene. Segmentation plays a vital role to extract information from an image to create homogenous regions by classifying pixels into groups thus forming regions of similarity. The homogenous regions formed as a result of segmentation indwell pixels having similarity in each region according to particular selection criteria e.g. Intensity, color etc. Image segmentation is the basic requirement of any computer vision application because people are generally interested only in certain parts of the image. Image segmentation results in non overlapping objects labeled with different region numbers. It should be noticed that no general technique has been developed yet to segment an image precisely, so different techniques are taking floor to perform segmentation. Threshold based image segmentation techniques discriminate regions on the basis of intensity value difference between pixels. Thresholds for image segmentation have been calculated based on maximum entropy, interclass variation, and histogram. The limitation of threshold based segmentation technique is that it performs well for images, which have only two components. For complex images, it is calculated to support further processes. Clustering is an approach in which pixels are classified to a cluster, which is closest among all clusters. Pixels having homogeneous characteristics belong to the same cluster and different with respect to pixels of other clusters. The pixels must follow the homogeneity criteria in the same cluster. In cluster based image segmentation techniques, it is necessary to choose a certain number of clusters initially which eventually reduces the dynamicity of the technique.

\section{LITRATURE REVIEW}

In this paper, attempt is made to overcome the problem which arises when image is segmented using both color and texture properties of the image. After pre-processing, the image is transformed from the RGB color space to $\mathrm{L}^{*} \mathrm{a} \mathrm{b}^{*}$ space. Then, the three channels of $\mathrm{L} * \mathrm{a}^{*} \mathrm{~b} *$ color space are separated and a single channel is selected depending upon the color under consideration. Next, Otsu based color segmentation is performed on the single channel image after which wavelet transform is applied to the image to obtain the particular object of interest [1].

In this paper, authors discussed about the difficulties which arises when attempt is made to extract the text objects from the PDF image. The problem arises due to variation in font size, font type, orientation and alignment. The enhancement is made in the two methods to solve the problems these methods are AC Coefficient based technique and Histogram Based Technique [2].

In this paper, new region growing color segmentation algorithm is presented. This algorithm is invariant to highlights and shading. This is accomplished in two steps. First, the average pixel intensity is removed from each RGB coordinate. This transformation mitigates the effects of highlights. Next, region seeds are obtained using the Mixture of Principal Components algorithm. Each region is characterized using two parameters. The first is the distance between the region prototype and the candidate pixel. The second is the 
distance between the candidate pixel and its nearest neighbour in the region. The inner vector product or vector angle is used as the similarity measure which makes both of these measures shading invariant [3].

In this paper, authors proposed methods for edge segmentation of satellite images. The various techniques are used for edge segmentation. These techniques are Sobel operator technique, Prewitt technique, Kiresh technique, Laplacian technique, Canny technique, Roberts technique and Edge Maximization Technique (EMT) [4]. These techniques are compared with one another to choose the best technique for edge segmentation. The selected best technique is applied to the satellite image for analysis.

In this paper, author presents a region growing technique for color image segmentation. The existing technique requires initials seeds which increase computation cost and execution time. The new technique is been proposed which reduces computation cost and execution time. This technique take initial seed as the central pixel of the image and grow region according to the grow formula [5].

\section{WATERSHED AND REGION GROWING TECHNIQUES}

In region growing technique the image is segmented into the similar areas on the basis of connected pixels values. Each of the pixels in a region is similar with respect to some characteristics or computed property such as color, intensity and/or texture. The region growing method is also classified as the pixel based image segmentation technique. In this method the initial seed is selected, on the basis of initial seed value it is decided that whether neighbour pixel is added to the region or not. The whole process of region growing algorithm is works in the form of iterations. When the whole process is completed clusters of the similar type of pixels is been formed. The region growing technique can correctly separate the regions that have the same properties. Region growing methods can provide the original images which have clear edges the good segmentation results. There are mainly three stages as indicated by figure 1 for watershed based image segmentation approach. The input image is first processed by the pre-processing stage and it is pass on to the watershed based image segmentation stage, the third and last stage is post-processing stage which process the image to remove the image over segmentation.

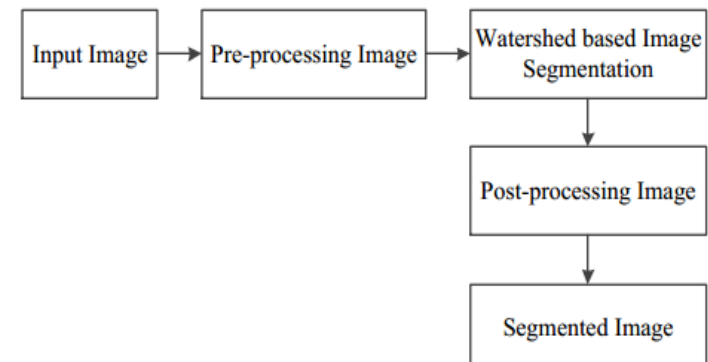

Fig 1: Block diagram of Watershed image segmentation Algorithm

\section{NEW PROPOSED ALGORITHM}

The new proposed algorithm is based on the image and color segmentation. The image and color segmentation is performed using the Ncut algorithm. In this algorithm, original image is converted to the brightness image. The converted image and original images are compared and matrix is made on the basis of Ncut eigenvectors and discrete segmentation

The degree of dissimilarity is calculated by the formula

$$
F(I)=\sqrt{R} \times \sum e_{i}^{2} / \sqrt{A_{i}}
$$

where, $I$ is the image to be segmented, $R$ total number of regions in the segmented image, Ai the area or the number of pixels of the Ith region and ei the color error of regions. ei is defined as the sum of the Euclidean distance of the color vectors between the original image and the segmented image of each pixel in the region. The term is a local measure which penalizes small regions or regions with a large color error. Ei indicates whether or not a region is assigned an appropriate feature (color). A large value of ei means that the feature of the region is not well captured during the SSF process In this paper, F is normalized by the size of the image and is scaled down by the factor $1 / 1000$. The smaller the value of $F$, the better is the segmentation result.

- Read image, convert into HSV

- Use the Gradient Magnitude as the Segmentation Function

- Result the Watershed Transform of the Segmentation Function

- Find region using numeration function then transform into gradient magnitude

- Seed function select for image segmentation process 
- Remove function call

- Final Result

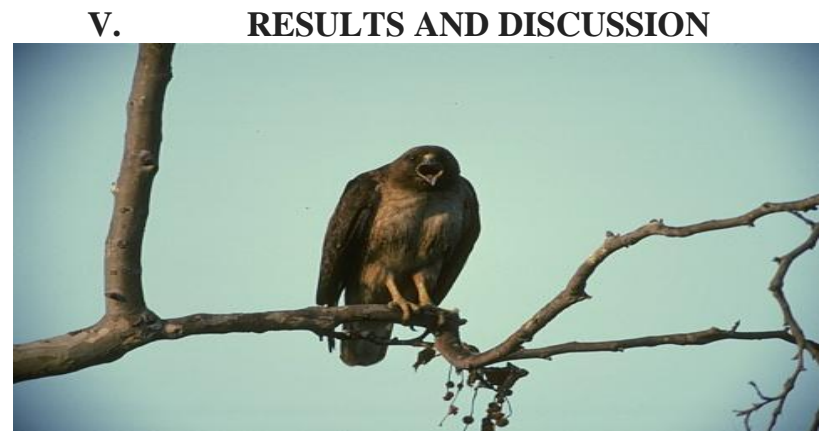

Fig1: Original Image

The original image is read and after watershed apply the result will be.

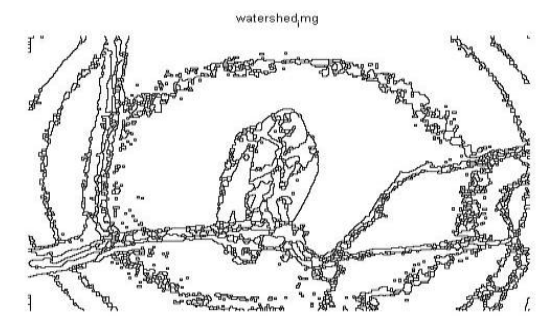

Fig2: watershed of the image

As, shown in the figure 2. The part of the image is show using improved watershed image.

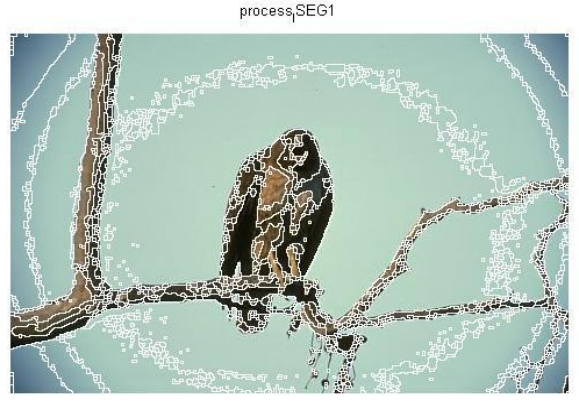

Fig3: The Selection seed of image segmented

As shown in figure 3, image is segmented through seed selection of image, it is easy to identify the segmented object.

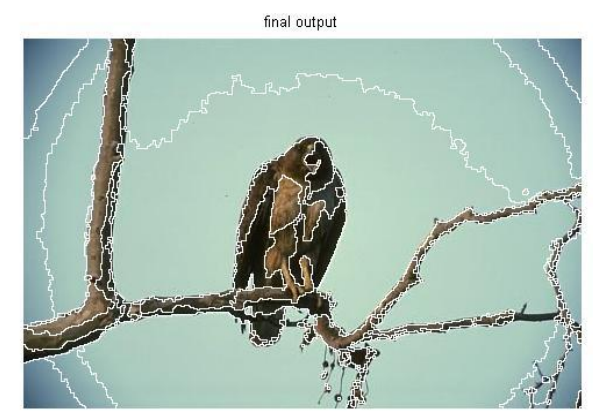

Fig4: The final output of region growing image segmentation

As shown in figure 4, image is segmented through and finally we get object in image either in background or in foreground. 


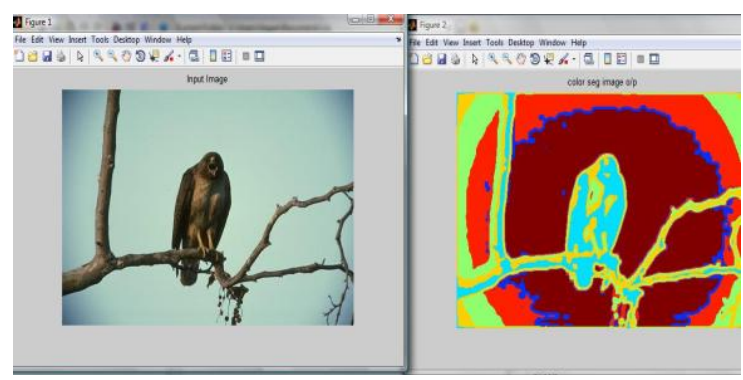

Fig 5: Color Segmentation

As, shown in the figure 5, the selected object on which image segmentation is performed, is now ready for the color segmentation. In the figure, color segmentation of the selected object is been shown.

We evaluate the Liu's F-factor (1) for all images for both the techniques. The results of comparison of the proposed method with the watershed technique are given in table (1). It is observed from table (1) that the Liu's F-Factor is lower for our method's results as compared to watershed segmentation.

Table(1) Liu's F-factor

\begin{tabular}{|l|l|l|l|l|}
\hline S.no & Image & $\begin{array}{l}\text { Using } \\
\text { watershed }\end{array}$ & Existing work & Present work \\
\hline 1. & Man and Building & $3.784 \mathrm{e}-004$ & $8.625 \mathrm{e}-006$ & $0.1816 \mathrm{e}-28$ \\
\hline 2. & Boat & $1.888 \mathrm{e}-004$ & $5.176 \mathrm{e}-006$ & $0.0730 \mathrm{e}-24$ \\
\hline 3. & Garden & $4.233 \mathrm{e}-004$ & $5.332 \mathrm{e}-006$ & $0.0521 \mathrm{e}-22$ \\
\hline 4. & Tree & $2.089 \mathrm{e}-004$ & $5.949 \mathrm{e}-006$ & $0.0010 \mathrm{e}-20$ \\
\hline 5. & Plane & $2.139 \mathrm{e}-004$ & $6.453 \mathrm{e}-006$ & $0.4079 \mathrm{e}-24$ \\
\hline 6. & Crow & $1.482 \mathrm{e}-004$ & $5.682 \mathrm{e}-006$ & $0.0702 \mathrm{e}-24$ \\
\hline 7. & Statues & $3.358 \mathrm{e}-004$ & $6.163 \mathrm{e}-006$ & $0.0602 \mathrm{e}-21$ \\
\hline
\end{tabular}

VI. CONCLUSION

In this paper, we conclude that old method of color image segmentation will do only color segmentation and having a more factor value, but we will improve first watershed than image segmentation or after that color segmentation done. Doing this compare the value of our algorithm having less than older algorithm, it's means our technique more efficient than older and in the older they will only do color segmentation or compare with watershed method in viewing, but we do firstly improve watershed than image segmentation after than color segmentation.

\section{REFERENCES}

[1] Amanpreet Kaur Bhogal et al," Color Image Segmentation Based On Color And Texture properties", INTERNATIONAL JOURNAL OF ADVANCED ENGINEERING SCIENCES AND TECHNOLOGIES Vol No. 8, Issue No. 2, $152-156$

[2] D.Sasirekha et al," Enhanced Techniques for PDF Image Segmentation and Text Extraction”, (IJCSIS) International Journal of Computer Science and Information Security, Vol. 10, No. 9, September 2012

[3] Slawo Wesolkowski and Paul Fieguth," Color Image Segmentation Using Vector Angle-Based Region Growing"

[4] Mr. Salem Saleh Al-amri et al," IMAGE SEGMENTATION BY USING EDGE DETECTION", International Journal on Computer Science and Engineering Vol. 02, No. 03, 2010, 804-807

[5] Om Prakash Verma et al," A Simple Single Seeded Region Growing Algorithm for Color Image Segmentation using Adaptive Thresholding", 2011 International Conference on Communication Systems and Network Technologies

[6] Haining Wang," Image Segmentation by Normalized Cut”, ECS 231 Final Project - Prof. Zhaojun Bai 